\title{
The gravitational-wave discovery space of pulsar timing arrays
}

\author{
Curt Cutler, ${ }^{1,2}$ Sarah Burke-Spolaor, ${ }^{1}$ Michele Vallisneri, ${ }^{1,2}$ Joseph Lazio, ${ }^{1}$ and Walid Majid ${ }^{1}$ \\ ${ }^{1}$ Jet Propulsion Laboratory, California Institute of Technology, \\ 4800 Oak Grove Drive, Pasadena, California 91109, USA \\ ${ }^{2}$ Theoretical Astrophysics, California Institute of Technology, Pasadena, California 91125, USA
}

(Received 11 September 2013; published 18 February 2014)

\begin{abstract}
Recent years have seen a burgeoning interest in using pulsar timing arrays (PTAs) as gravitational-wave (GW) detectors. To date, that interest has focused mainly on three particularly promising source types: supermassive black hole binaries, cosmic strings, and the stochastic background from early-Universe phase transitions. In this paper, by contrast, our aim is to investigate the PTA potential for discovering unanticipated sources. We derive significant constraints on the available discovery space based solely on energetic and statistical considerations: we show that a PTA detection of GWs at frequencies above $\sim 10^{-5} \mathrm{~Hz}$ would either be an extraordinary coincidence or violate "cherished beliefs;" we show that for PTAs GW memory can be more detectable than direct GWs, and that, as we consider events at ever higher redshift, the memory effect increasingly dominates an event's total signal-to-noise ratio. The paper includes also a simple analysis of the effects of pulsar red noise in PTA searches, and a demonstration that the effects of periodic GWs in the $\sim 10^{-7}-10^{-4.5} \mathrm{~Hz}$ band would not be degenerate with small errors in standard pulsar parameters (except in a few narrow bands).
\end{abstract}

DOI: $10.1103 /$ PhysRevD.89.042003

PACS numbers: 04.80.Nn, 04.30.-w, 95.85.Sz, 97.60.Gb

\section{INTRODUCTION}

The idea of detecting gravitational waves (GWs) by monitoring the arrival times of radio pulses from neutron stars (i.e., by pulsar timing) was first proposed by Sazhin [1] and Detweiler [2]; its modern formulation by Hellings and Downs [3] emphasizes the importance of searching for correlations in the pulse-timing time deviations among an array of intrinsically stable millisecond pulsars. The last few years have seen a strong renewed interest in these searches, with the formation of three major pulsar timing programs: the European Pulsar Timing Array (EPTA, [4,5]), the North American Nanohertz Observatory for Gravitational Waves (NANOGrav, [6,7]), and the Australian Parkes Pulsar Timing Array (PPTA, [8,9]), which have now joined into a global collaboration, the International Pulsar Timing Array (IPTA, [10,11]).

The most promising known sources of GWs for PTAs are inspiraling supermassive black hole binaries (SMBHBs). Some estimates suggest that these will be detected by PTAs as soon as 2016-2020 [12,13]. The first detection could plausibly identify the inspiral waves from an orbiting SMBHB (see, e.,g., [14-16]), the burst waves that follow its coalescence $[17,18]$ or a stochastic background from many SMBHBs (see, e.,g., $[19,12])$. Pulsar timing already provides the most stringent upper limit on $\Omega_{\mathrm{GW}} \equiv \rho_{\mathrm{GW}} / \rho_{0}$ (the ratio of the energy density in GWs to the closure density of the Universe): $\Omega_{\mathrm{GW}}<1.3 \times 10^{-9}$ at $f=2.8 \times 10^{-9} \mathrm{~Hz}$ [20]. This limit is beginning to impact standard theories of hierarchical structure formation via constraints on the SMBH merger rate.
In this article we explore the discovery potential of PTAs. Our main motivation is to minimize the risk that current observing strategies and planned data-analysis pipelines artificially preclude the discovery of various types of sources. For instance, most pulsars in PTAs are currently observed with irregular cadences of $\sim 2-4$ weeks. The observational strategies for most pulsar timing arrays are currently optimized to be sensitive to the gravitational wave background (based on strategies as determined by [21]). This is appropriate for GWs at the lowest observable frequencies (of order the inverse of the total observation time, $\sim 3 \times 10^{-9} \mathrm{~Hz}$ ), where PTAs are particularly sensitive. However a search for GW bursts lasting (say) $10^{5} \mathrm{~s}$ would clearly benefit greatly from coordinated timing observations (using a few radio telescopes) that get repeated several times a day. Thus we address the following questions:

(i) Is there a strong motivation for increasing the observing cadence to improve our sensitivity to GWs with frequencies $\sim 10^{-6}-10^{-5} \mathrm{~Hz}$ ?

(ii) What constraints can we impose on the PTA discovery space based on simple energetic, statistical, and causality arguments?

In addressing the first question, an important issue that arises is whether, even if strong sources exist in this band, our sensitivity might be degraded by degeneracies between GW effects and small errors in the timing-model parameters of the monitored pulsars. In addressing the second question, we are necessarily retracing some of the trails blazed by Zimmermann and Thorne [22] (hereinafter ZT82) in their classic paper, "The gravitational waves that bathe the Earth: upper limits based on theorists' cherished 
beliefs." However there are important differences between our paper and theirs:

(i) ZT82 restricted attention to sources at $z \lesssim 3$, while we consider the case of very high- $z$ sources as well.

(ii) Unlike ZT82, we include the "memory effect" among potential observables; its detection turns out to be especially promising in the high- $z$ case.

(iii) ZT82 restricted attention to GWs in the frequency range $10^{-4}<f<10^{4} \mathrm{~Hz}$ (the band of interest for ground-based and space-based interferometers), while we focus on GWs with $f \lesssim 10^{-5} \mathrm{~Hz}$. (However, there are several instances for which the ZT82 estimates extend trivially to lower frequency; we will note these instances in our paper as they arise.)

This paper is organized as follows. In Sec. II we describe a simple general framework for thinking about pulsar timing observations, and we characterize how the detection signal-to-noise ratio (SNR) scales with quantities such as the number of pulsars surveyed, the timing accuracy provided by each pulsar observation, the observing cadence, and the total observation time. We also briefly review pulsar timing noise, with some emphasis on its red-noise component. In Sec. III we summarize salient results regarding PTA searches for SMBHBs and cosmic strings, largely to provide points of comparison with possible unknown GW sources. In Sec. IV we demonstrate that the timing residual signatures of GWs in the $10^{-7}-10^{-4.5} \mathrm{~Hz}$ band are not degenerate with small errors in the pulsar parameters, except for very narrow frequency bands; had this been otherwise, there would have been little point in considering more fundamental constraints on possible sources in this band. In Secs. V and VI we investigate what constraints on source strengths arise from fundamental considerations of energetics, statistics, and causality. In Sec. VII we discuss how our estimates get modified for highly beamed sources, and for sources in our Galaxy. In Sec. VIII we summarize our conclusions, listing some caveats.

Regarding notation, we adopt units in which $G=c=1$. Also, the signal frequency $f$, observation time $T_{\mathrm{obs}}$, and signal duration $T_{\text {sig }}$ all refer to time as measured in the observer's frame, at the Solar System barycenter.

\section{THE PTA SIGNAL-TO-NOISE RATIO FOR GW SIGNALS OF KNOWN SHAPE}

\section{A. Signal-to-noise ratio for white-noise signals}

In the rest of this paper, we are going to assume an idealized, general scaling law for the detection SNR of an individual GW source, as observed by a pulsar timing array: to wit,

$$
\mathrm{SNR}^{2}=M N\left\langle\frac{\delta t_{\mathrm{GW}}^{2}}{\delta t_{\text {noise }}^{2}}\right\rangle
$$

where

(i) $\delta t_{\mathrm{GW}}$ is the timing residual due to GWs; (ii) $\delta t_{\text {noise }}$ is the noise in the residuals, which includes contributions from the observatory, from pulse propagation, and from intrinsic pulsar processes;

(iii) $\langle\cdots\rangle$ denotes the average over all pulsars in the PTA and over all observed pulses;

(iv) $M$ is the number of pulsars in the PTA; and.

(v) $N$ is the total number of observations for each pulsar. In what follows, purely for simplicity we will assume that $\delta t_{\mathrm{rms}}$ is roughly the same across PTA pulsars and observations, so we define

$$
\left\langle\frac{\delta t_{\mathrm{GW}}^{2}}{\delta t_{\mathrm{rms}}^{2}}\right\rangle=\frac{\left\langle\delta t_{\mathrm{GW}}^{2}\right\rangle}{\delta t_{\mathrm{rms}}^{2}}
$$

with $\delta t_{\text {rms }}$ a representative rms value for the noise.

The term "timing residual" requires definition: it is the difference between the time of arrival (TOA) of a train of pulses observed at the radio telescope and the TOA predicted by the best-fitting timing model for a pulsar. This deterministic model includes parameters (such as the sky position of and distance to the pulsar) that affect the propagation of signals to the observatory, as well as parameters (such as the pulsar period and its derivatives and, if needed, orbital elements for pulsars in binaries) that describe the intrinsic time evolution of the pulsar's emission.

The pulses from millisecond pulsars are usually too weak to be observed individually, so the TOAs refer to integrated pulse profiles obtained by "folding" the output of radiometers with the putative pulsar period over observations with durations of tens of minutes to an hour. Typically, such pulsar timing observations are repeated at intervals of two to four weeks, yielding sparse data sets; however, the individual observations are often run quasisimultaneously at multiple receiving frequencies (typically one hour to two days apart, since the feeds need to be switched), yielding a set of TOAs at the same epoch. See $[23,24]$ and references therein for more detail.

In analogy with other applications in GW data analysis [25], our scaling for the SNR can be motivated by considering a ratio of likelihoods: namely, the likelihood of the residual data $r_{i}$ (with $i$ indexing both epochs and pulsars) under the hypothesis that $r_{i}=g_{i}+n_{i}$, with $g_{i}$ describing a GW signal of known shape, and $n_{i}$ denoting noise; and the likelihood of the residuals under the noise-only hypothesis $r_{i}=n_{i}$. For Gaussian noise, when the GW signal is really present, the likelihood ratio is

$$
\exp \left\{g_{i}\left(C^{-1}\right)^{i j} g_{j} / 2+n_{i}\left(C^{-1}\right)^{i j} g_{j}\right\}
$$

(summation over the indices $i$ and $j$ is implied), where $C_{i j}=\left\langle n_{i} n_{j}\right\rangle$ is the variance-covariance matrix for the noise. The first term in the exponent, which depends only on the GWs, is identified as $\mathrm{SNR}^{2} / 2$, while the second term is a random variable with mean zero and variance (over noise realizations) equal to $\mathrm{SNR}^{2}$. This can be proved, e.g., by considering that Gaussian noise with covariance $C$ can be written as $\left(C^{1 / 2}\right) \bar{n}$, with $\left(C^{1 / 2}\right)\left(C^{1 / 2}\right)^{T}=C$ the 
Cholesky decomposition of $C$, and with $\bar{n}$ a vector of uncorrelated, zero-mean/unit-variance Gaussian variables. Then

$$
\begin{aligned}
& \left\langle\left(n_{i}\left(C^{-1}\right)^{i j} g_{j}\right)\left(n_{l}\left(C^{-1}\right)^{l m} g_{m}\right)\right\rangle \\
& \quad=\left(C^{-1}\right)^{i j} g_{j}\left(C^{1 / 2}\right)_{i}^{k}\left\langle\bar{n}_{k} \bar{n}_{p}\right\rangle\left(C^{1 / 2}\right)_{l}^{p}\left(C^{-1}\right)^{l m} g_{m} \\
& \quad=\left(C^{-1}\right)^{i j} C_{i l}\left(C^{-1}\right)^{l m} g_{j} g_{m}=\left(C^{-1}\right)^{j m} g_{j} g_{m} .
\end{aligned}
$$

Equation (2.1) follows immediately under the (strong) assumption that noise is uncorrelated and homogenous among pulsars and epochs, so that it can be represented by $\left(C^{-1}\right)^{i j}=\delta^{i j} / \delta t_{\mathrm{rms}}^{2}$. We are assuming also that the sampling of pulsars and epochs in the data set is sufficiently broad and nonpathological that $\sum_{i} g_{i}^{2} \simeq M N\left\langle\delta t_{\mathrm{GW}}^{2}\right\rangle$; that is, that the sampling can effectively perform an average over time and pulsar sky position. If the noise is uncorrelated (i.e., white), but not homogeneous, Eq. (2.1) still stands, provided that $\delta t_{\mathrm{rms}}^{2}$ can be taken to represent a suitable averaged noise.

Under these assumptions, Eq. (2.1) is remarkable in that the actual form of the signal to be detected appears only through its variance $\left\langle\delta t_{\mathrm{GW}}^{2}\right\rangle$, and that the structure of observations appears only through their overall number $M \times N$ and rms noise $\delta t_{\mathrm{rms}}^{2}$. By contrast, one may have imagined that detecting (say) quasisinusoidal signals of high frequency $f_{\mathrm{GW}}$ would require rapid-cadence observations spaced by $\Delta t \lesssim 1 / f_{\mathrm{GW}}$, according to the Nyquist theorem. However, that theorem is a statement about the reconstruction of the whole of a function on the basis of a set of regularly spaced samples, but it does not apply to our casecomputing the likelihood that a signal of known shape is present in the data [26]. In effect, we are checking that the measured data are consistent with our postulated signal: for uncorrelated noise, it does not matter when we check, but only how many times we do it.

\section{B. Relaxing the assumption of white noise}

There are two important considerations that challenge our assumption of white, uncorrelated noise.

The first is that the residuals include a stochastic contribution due to the over-fitting of noise (and possible GWs) at the time of deriving the timing model. We discuss this further in Sec. IV, where we show empirically that the detection of quasisinusoidal signals at most frequencies would not be affected. From a formal standpoint, van Haasteren and colleagues [27] show that it possible to marginalize the likelihood over timing-model parameter errors $\delta \xi$ by replacing the inverse covariance in Eq. (2.3) with $C^{-1}-C^{-1} M\left(M^{T} C^{-1} M\right)^{-1} M^{T} C^{-1}$, where $M$ is the design matrix for the timing-model fit, so that the extra contribution to the residuals has the form $M \delta \xi$. (A similar strategy of "projecting out" parameter errors was employed earlier by Cutler and Harms [28], in the context of removing residual noise from slightly incorrect GW foreground subtraction.) For uncorrelated noise, Eq. (2.1) is modified only by restricting the computation of $\left\langle\delta t_{\mathrm{GW}}^{2}\right\rangle$ to the $\mathrm{GW}$ components that are not absorbed away by the timing model (and this is indeed what we investigate in Sec. IV).

The second important consideration (and for which the $\mathrm{GW}$ frequency does matter) is the impact of correlated noise. The physically interesting case here is that of long-term correlations, which generate red noise that is stronger at low frequencies. To understand the impact of red noise, we study a toy model in which the $N$ observations are organized in $P$ "clumps" of $Q$ TOAs taken at nearby times (with $N=P \times Q$ ), and where noise consists of two components: uncorrelated noise with variance $\sigma^{2}$ and noise with variance $\kappa^{2}$ that is completely correlated within clumps, and completely uncorrelated between clumps. (We use $\kappa$ since

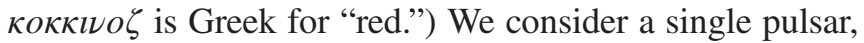
although the generalization to more is trivial.

The resulting $C$ has the structure

$$
C=\sigma^{2} I+\kappa^{2} \sum_{i=1}^{P} O_{i},
$$

where each $O_{i}$ is a matrix that has ones for every component corresponding to a combination of samples in the same burst, and zeros everywhere else. Each $O_{i}$ can also be written as $u_{i} u_{i}^{T}$, where $u_{i}$ is a vector that has ones for the components in clump $i$, and zeros everywhere else. From the block structure of $C$ and the Woodbury lemma [29], it follows that

$$
C^{-1}=\sigma^{-2} I-\frac{\sigma^{-2}}{P+\kappa^{-2} / \sigma^{-2}} \sum_{i=1}^{P} O_{i}
$$

If the characteristic frequency of the GW signal is "slower" than the timescale of a clump (i.e., the time over which the $Q$ samples in a clump are collected), then the sum $\sum_{i} g_{i}^{T} O_{i} g_{i} \simeq P Q^{2}\left\langle\delta t_{\mathrm{GW}}^{2}\right\rangle$, because the same value of $g$ is being summed over and over in each burst. It follows that

$$
\mathrm{SNR}^{2}=\frac{\left\langle\delta t_{\mathrm{GW}}^{2}\right\rangle P Q}{\sigma^{2}+Q \kappa^{2}}=\frac{\left\langle\delta t_{\mathrm{GW}}^{2}\right\rangle P}{\sigma^{2} / Q+\kappa^{2}}
$$

that is, the repeated observations in each clump average out the uncorrelated component of noise (as $\propto 1 / \sqrt{Q}$ ), but not its correlated part. Increasing the number of observations in a clump provides diminishing returns as $\sigma^{2} / Q \rightarrow \kappa^{2}$.

Let us follow the other branch of our derivation: if the characteristic frequency of the GW signal is "faster" than the timescale of the clumps, then, barring special coincidences, $\sum_{i} g_{i}^{T} O_{i} g_{i} \simeq P Q\left\langle\delta t_{\mathrm{GW}}^{2}\right\rangle$, and $\mathrm{SNR}^{2}$ reduces (modulo an $O[1 / Q]$ correction) to the general expression (2.1), with $N=P Q$.

\section{Noise characteristics inferred from observational data}

In this section, we consider the characteristics of noise for real pulsars. Namely, to what extent is our analysis applicable to timing residuals from actual PTAs? 
For the radiometer noise due to thermal effects in the receiving system, the assumption of no correlations (i.e., "white") is well justified: for observations over a radio frequency bandwidth $\Delta \nu$, the correlation timescale is $(\Delta \nu)^{-1}$, so this noise contribution is effectively uncorrelated in time. Further, from thermodynamic considerations, the assumption of Gaussianity is also well justified.

Pulsars can show correlated, red-spectrum fluctuations in their TOAs, and Cordes and Shannon [30] present a summary of various effects, ranging from intrinsic spin fluctuations to magnetospheric and propagation effects; see also [31]. These effects have spectral densities $\propto f^{-x}$, with $x$ typically $>1$ and in some cases $>4$. On timescales $\sim 5$ years $\left(f \sim 10^{-8.2} \mathrm{~Hz}\right)$, the residuals appear to be dominated by white components ([6,32]; see also Figs. 10 and 11 of [8] for a visual representation of noise effects in PPTA pulsars). Even if $\sigma^{2} \approx \kappa^{2}$ at frequencies $\sim 10^{-8.2} \mathrm{~Hz}$, at higher frequencies $\left(\gtrsim 10^{-7} \mathrm{~Hz}\right)$, the variance from white processes will exceed that of any red processes with relatively shallow spectra $(x \approx 1)$ by a factor of approximately 15 ; for red processes with steeper spectra $(x \approx 4)$, the ratio will be even larger.

In our toy model, the red-noise component of the variance is amplified by the clump multiplicity $Q$ [Eq. (2.7)]. For more general observation schemes and red-noise processes, we may think of the number of clumps $P$ as $T_{\text {obs }} / T_{\text {red }}$, where $T_{\text {obs }}$ is the total duration of observation, and $T_{\text {red }}$ is the correlation timescale of the most significant red-noise process; then $Q \simeq N\left(T_{\text {red }} / T_{\text {obs }}\right)$. For GW signals with frequency $\lesssim 1 / T_{\text {red }}$, our toy model would then suggest that

$$
\mathrm{SNR}^{2}=\frac{\left\langle\delta t_{\mathrm{GW}}^{2}\right\rangle}{\sigma^{2} / N+\kappa^{2}\left(T_{\mathrm{red}} / T_{\mathrm{obs}}\right)} ;
$$

that is, the $1 / \sqrt{N}$ averaging of noise becomes limited by red noise once $N \sim\left(\sigma^{2} / \kappa^{2}\right) \times\left(T_{\text {obs }} / T_{\text {red }}\right)$-an interesting scaling in its own right. For GW signals with frequencies $\gtrsim 1 / T_{\text {red }}$, the simpler scaling (2.1) applies. (In [13], Siemens et al. carry out a related analysis for the detectability of the stochastic background.)

In the remainder of this paper, we neglect the effects of red noise in the scaling of SNR and assume the expression of Eq. (2.1). Our assumption is correct because one or more of the following circumstances will be true (or true enough) in practice:

(i) The characteristic GW frequency of interest will be greater than $1 / T_{\text {red }}$ for the most significant red-noise component.

(ii) For a majority of the pulsars in the PTA, the whitenoise variance will exceed that of the most dominant red-noise process for the time scales of interest.

(iii) The number of observations will not saturate the averaging of white noise with respect to subdominant red noise (i.e., in the "clump" picture, $\sigma^{2} / Q>\kappa^{2}$ ).

\section{BRIEF REVIEW OF PROSPECTS FOR PTA SEARCHES OF SUPERMASSIVE BLACK HOLE BINARIES AND COSMIC STRINGS}

Here we collect a few salient points concerning PTA searches for SMBHBs and cosmic strings, mostly to provide points of comparison with the hypothetical sources we consider in the next sections. We refer the reader to the literature cited below for more details.

\section{A. The detectability of GWs from supermassive black hole binaries}

When two galaxies merge, the SMBHs at their centers are brought together by tidal friction from the surrounding stars and gas. It seems likely that their separation eventually shrinks to the point at which gravitational radiation emission dominates the inspiral, and the two SMBHs eventually coalesce [33]. The GWs from all inspiraling SMBHBs in the observable Universe contribute to a stochastic background of GWs with characteristic amplitude $h_{c} \sim h_{\mathrm{rms}} \sqrt{f}$ given by

$$
h_{c} \approx A\left(f / f_{0}\right)^{-\beta}
$$

in the PTA band, where $\beta \approx 2 / 3$ and $A$ is predicted to be in the range $5 \times 10^{-16}-5 \times 10^{-15}$ for $f_{0}=10^{-8} \mathrm{~Hz}$ $[12,19,34,35]$. Depending on the actual $A$, the first PTA detection of GWs is expected between 2016 and 2020 [13].

The background is expected to be dominated by binaries with chirp masses $M_{c} \equiv\left(m_{1} m_{2}\right)^{3 / 5}\left(m_{1}+m_{2}\right)^{-1 / 5} \sim$ $10^{8} M_{\odot}$ at $z \lesssim 2$. At frequencies above $f \approx 10^{-8} \mathrm{~Hz}$, sources are sparse enough that the central limit theorem does not apply, so the distribution is significantly nonGaussian and a few brightest sources would appear above the background. Thus, the first PTA discovery could either be an individual strong (and possibly nearby) source, or the full background.

\section{B. The detectability of GWs from cosmic strings}

There are several mechanisms by which an observable network of cosmic (super)strings could have formed in the early Universe [36]. Simulations have shown that string networks rapidly approach an attractor: the distribution of straight strings and loops in a Hubble volume becomes independent of initial conditions. The network properties $d o$ depend on two fundamental parameters: the string tension $\mu$ and the string reconnection probability $p$. The size of string loops at their birth should in principle be derivable from $\mu$ and $p$, but the studies are difficult and different simulations have produced very different answers. Therefore most astrophysical analyses today assume that the size of loops at their birth can be parametrized as $\alpha H^{-1}(z)$, where $H^{-1}(z)$ is the Hubble scale when the loop is "born," and where $\alpha$ is treated as a third unknown parameter. We refer the reader to $[36,37]$ for nice reviews. To make matters 
more complicated, Polchinski has argued that the distribution of loop size at birth is actually bimodal, with both relatively large and small loops being produced at the same epoch [38]. Regarding the string tension $\mu$, physically motivated values range over at least 6 orders of magnitude: $10^{-12} \lesssim \mu \lesssim 10^{-6}$.

Once formed, string loops oscillate and therefore lose energy and shrink due to GW emission. These waves form a stochastic GW background. In addition to this approximately Gaussian background, the cusps and kinks that form on the string loops emit highly beamed GW bursts $[39,40]$. Depending on the string parameters, PTAs could discover the stochastic background, the individual bursts, both, or neither. The limit that $\Omega_{\mathrm{GW}}\left(f \sim 1 \mathrm{yr}^{-1}\right) \lesssim 1 \times 10^{-8}$, from pulsar timing [4], corresponds to a limit on the string tension of $G \mu \leq 4.0 \times 10^{-9}$. (An even more stringent limit on $\Omega_{\mathrm{GW}}$ was published very recently [20].)

\section{Current constraints on $\Omega_{\mathrm{GW}}(f)$}

As mentioned, the current limit on $\Omega_{\mathrm{GW}}(f)$ from pulsar timing is $\Omega_{\mathrm{GW}}<1.3 \times 10^{-9}$ at $f=2.8 \times 10^{-9} \mathrm{~Hz}$ [20]. By comparison, the limit from first-generation ground-based interferometers is $\Omega_{\mathrm{GW}}(f \sim 100 \mathrm{~Hz})<6.9 \times 10^{-6}$ [41].

From Big Bang nucleosynthesis, we know also that any GW stochastic background that existed already when the Universe was three minutes old satisfies $\Omega_{\mathrm{GW}}<$ $1.5 \times 10^{-5}$ today [42]. Combined measurements of CMB angular power spectra (which are sensitive to lensing by a stochastic GW background) with matter power spectra also yield $\Omega_{\mathrm{GW}} \lesssim 10^{-5}$ today, but this method is sensitive to any GWs produced before recombination at $z \approx 1100$ [43]. For GWs generated in the low- $z$ Universe, combining results from Planck, WMAP, SDSS, and $H_{0}$ measurements gives the limit $\Omega_{\mathrm{GW}} \lesssim 6 \times 10^{-3}$ [44].

\section{SPECTRAL ABSORPTION EFFECTS FROM PULSAR TIMING-MODEL FITTING}

The best knowledge of pulsar parameters comes from the iterative observation and refinement of a timing model, which predicts the times of arrival of all the pulses as a function of all relevant parameters, such as the period and period derivatives of the pulsar's intrinsic spin; the position, proper motion, and parallax of the pulsar; and possibly parameters that describe the motion of the pulsar in a binary system. Depending on the cadence and total time of observation, and on the shape and duration of the GWs, the effects of the GWs on pulse arrival times may correlate with the effects of changing the pulsar parameters, so the GW power may be partly or entirely absorbed by the parameter-fitting process (see, e.g., the study of the effect of a GW background on pulsar timing parameter estimation [45]).

As a specific study of this effect, here we investigate the absorption of sinusoidal GWs to demonstrate frequencydependent signal loss to pulsar parameter fitting. To do this, we use the Tempo 2 software suite [46] to simulate a set of timing residuals for pulsar J0613-0200 [8], as observed with the Parkes observatory. We generate one TOA every other day for $T_{\text {obs }}=1,000$ days, at a random time compatible with the pulsar being visible from the observatory, and we add a white-noise component with rms amplitude of $100 \mathrm{~ns}$. Into these simulated residuals we inject sinusoidal GWs from a circular SMBHB binary located at $\mathrm{RA}=\mathrm{Dec}=0$, varying the GW frequency $f$ between $10^{-7.95}$ and $10^{-4.5} \mathrm{~Hz}$ (corresponding to $\mathrm{GW}$ periods of $\sim 1,000$ days to eight hours), and setting $h_{+}=h_{\times}=10^{-3} f$, so that the SNR is fixed.

For each GW frequency, we measure the power spectral density of the relevant frequency component before the timing-model fit and after seven different types of fit: a fit against the full set of parameters and individual fits for pulsar frequency, frequency derivative, position, proper motion, parallax, and binary period. Figure 1 shows the ratio of the power spectral densities in each case, as a function of the source GW frequency. In effect, we are showing the absorption spectrum of sinusoidal GWs, as filtered by the timing-model fit. Above $10^{-7} \mathrm{~Hz}, \gtrsim 95 \%$ of the signal is preserved even in a full parameter fit, with only narrow absorption features. It is clear that most of these features are specific to this pulsar's binary orbit (and its harmonics), and would not appear at the same frequencies for other pulsars in a PTA.

However, absorption features originating from nonbinary parameters will occur in all pulsars. Specifically, absorption at $f=1 /$ year (corresponding to pulsar position/proper motion) and 1/6 months (corresponding to parallax) can result in up to $100 \%$ loss of the GW signal. Similarly, as the GW period approaches the total duration of pulsar observations, fitting the pulsar spin frequency and frequency derivative results in significant signal absorption. The sensitivity to GWs at these lower frequencies would be better in a longer data set (see, e.g., the low-frequency sensitivity curves in [15]).

At high frequencies, only two narrow absorption features may be common across a PTA: these correspond to the observing cadence [here at $1 /(2$ days $)=10^{-5.238} \mathrm{~Hz}$, and to the sidereal day [at $1 /(23.934 \mathrm{hr}) \simeq 10^{-4.935} \mathrm{~Hz}$ ]. The former can be avoided with higher-cadence or irregular observations, but the latter reflects the limitations of using a single observatory, which can only observe a source while it is above the horizon. In our simulation we have chosen random observation times within the window of coverage, but more structured observing cycles can engender even deeper features. By contrast, this feature can be avoided for a polar target that never sets.

To summarize, our example study suggests that for a majority of PTAs a high-frequency GW signal will be well preserved through the standard timing-model fitting process, save for narrow features at roughly the observing cadence and the sidereal day. GWs at frequencies close 


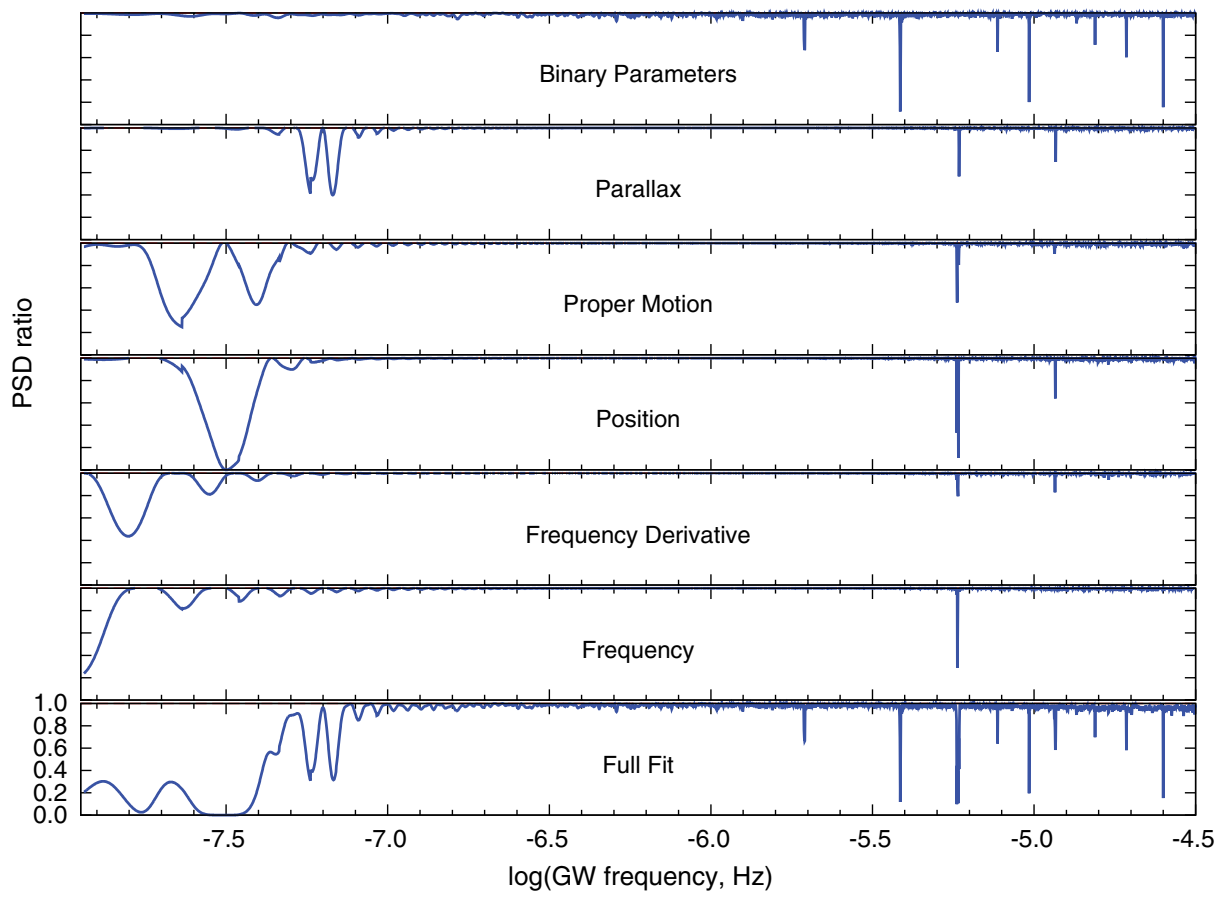

FIG. 1 (color online). GW power absorbed by fitting for various pulsar parameters as a function of GW frequency, for pulsar J0613-0200. "PSD ratio" refers to the pre-fit power spectral density value for the given frequency, divided by its post-fit value. All simulated GWs were sinusoids at the given GW frequency. For each panel, only the indicated parameters were used for fitting, while the other parameters were held fixed at the values given in [8]. At high frequencies, only narrow features are evident (mostly due to fitting of the pulsar's binary motions), but low-frequency GW signals are significantly absorbed by standard fitting parameters.

to either $(1 \text { year })^{-1}$ or $(6 \text { months })^{-1}$ will be significantly impacted, as will GWs with periods approaching the longest-duration pulsar observations.

\section{DISCOVERY SPACE FOR SOURCES IN THE LOW-REDSHIFT UNIVERSE, $z \lesssim O(1)$}

In this section we begin to characterize the PTA discovery space for the case of sources in the low-redshift Universe, by which we mean $z \lesssim O(1)$. We imagine that there is some heretofore undiscovered GW source, and we ask what it would take for it to be detectable via pulsar timing. We consider separately the case of modeled signals (for sources already conceived by theorists, so that a parametrized waveform model can be used in a matchedfiltering search), the case of unmodeled bursts, and the case of the gravitational memory effect from modeled sources.

We will assume that the GW sources are distributed isotropically and that we do not occupy a preferred location in space and time with respect to them - that is, we assume that the Earth is not improbably close (spatially) to one of the sources, and that the sources have been emitting GWs for a significant fraction of the last $10^{10}$ years.

We parametrize our projections in terms of the energy density $\Omega_{\mathrm{GW}}$. Because we consider sources in the lowredshift Universe, in what follows we ignore redshift effects. Nevertheless our results at $z \sim 1$ match on nicely to our results for high $z$ in Sec. VII.

\section{A. Discovery space for modeled GW signals in the low-redshift Universe}

As we established in Sec. II, the SNR modeled GW signals as observed by a PTA is

$$
\mathrm{SNR}^{2}=\frac{\left\langle\delta t_{\mathrm{GW}}^{2}\right\rangle}{\delta t_{\mathrm{rms}}^{2}} M N=\frac{\left\langle\delta t_{\mathrm{GW}}^{2}\right\rangle}{\delta t_{\mathrm{rms}}^{2}} M p \min \left\{T_{\mathrm{sig}}, T_{\mathrm{obs}}\right\},
$$

where $\left\langle\delta t_{\mathrm{GW}}^{2}\right\rangle$ and $\delta t_{\mathrm{rms}}^{2}$ are the mean-square-averaged timing residuals due to GWs and measurement/pulsar noise; $M$ is the number of pulsars in the array; $N$ is the number of times each pulsar is observed, which we rewrite in terms of the cadence of observation $p$ (e.g., 1/day), the total duration of observation $T_{\text {obs }}$ (e.g., 3 years), and the typical duration of the GW signal $T_{\text {sig. }}$.

For a sinusoidal GW signal of frequency $f$ and rms amplitude at Earth $h=\sqrt{h_{+}^{2}+h_{\times}^{2}}$, the root-mean-square timing residual averages ${ }^{1}$ to

$$
\bar{\delta} t_{\mathrm{GW}} \equiv \sqrt{\left\langle\delta t_{\mathrm{GW}}^{2}\right\rangle}=\frac{1}{4 \sqrt{3} \pi} \frac{h}{f} \simeq \frac{1}{20} \frac{h}{f} .
$$

\footnotetext{
${ }^{1}$ To derive Eq. (5.2) we compute the Estabrook-Wahquist [47] fractional Doppler response (for the pulsar "Earth term" alone) to a sinusoidal GWs given by $h_{+}(t)+i h_{\times}(t)=(h / \sqrt{2}) \exp 2 \pi \mathrm{i} f t$, take the antiderivative to obtain the corresponding pulse-time delay, square and average over time, sky position, and polarization angle.
} 
Furthermore, the average rate at which the sources radiates energy in GWs is $\dot{E}=\left(\pi^{2} / 2\right) h^{2} f^{2} d^{2} \simeq 5 h^{2} f^{2} d^{2} \quad[25$, Eq. (1.160)], where $d$ is the distance to the source, and $G=c=1$ (as we will set throughout). The GW energy density from source of this kind is

$$
\Omega_{\mathrm{GW}} \rho_{0} \simeq\left(\dot{E} T_{\mathrm{sig}}\right)\left(R_{4} \tau_{0}\right)
$$

where $R_{4}$ is the spacetime rate density of sources, and $\tau_{0} \sim$ $10^{10} \mathrm{yr}$ is the current age of the Universe. Approximating the closure density $\rho_{0}=3 H^{2} / 8 \pi$ as $\tau_{0}^{-2} / 10$ (since $\tau_{0} \simeq H^{-1}$ ) and rewriting $R_{4} \equiv\left(V_{R} T_{R}\right)^{-1}$ in terms of a fiducial volume $V_{R}$ and the total event rate $T_{R}$ in that volume, we can reexpress the expected GW-induced timing residual as

$$
\bar{\delta} t_{\mathrm{GW}} \simeq \frac{1}{150} f^{-2} d^{-1}\left(\frac{\Omega_{\mathrm{GW}} V_{R} T_{R}}{\tau_{0}^{3} T_{\mathrm{sig}}}\right)^{1 / 2} .
$$

We estimate the distance to the closest source that would be observed over time $T_{\text {obs }}$ by setting

$$
\frac{4}{3} \pi d^{3} \max \left\{T_{\text {obs }}, T_{\text {sig }}\right\} R_{4}=1
$$

(where the maximum accounts for the persistence of multiple emitting sources if $T_{\text {sig }}>T_{\text {obs }}$ ), whence

$$
d_{\text {near }} \simeq\left[\frac{3}{4 \pi} \frac{V_{R} T_{R}}{T_{\text {sig }}} \min \left\{1, T_{\text {sig }} / T_{\text {obs }}\right\}\right]^{1 / 3} .
$$

Folding together all the results of this section, we obtain the corresponding, largest SNR that would be observed as

$$
\begin{aligned}
\mathrm{SNR}_{\text {near }}^{2} & \simeq 10^{-4} \frac{\Omega_{\mathrm{GW}}}{f^{4} \tau_{0}^{3}} \frac{M p T_{\mathrm{obs}}}{\delta t_{\mathrm{rms}}^{2}}\left[\frac{V_{R} T_{R}}{T_{\mathrm{sig}}} \min \left\{1, \frac{T_{\mathrm{sig}}}{T_{\mathrm{obs}}}\right\}\right]^{1 / 3} \\
& \simeq 2 \times 10^{-4} \frac{\Omega_{\mathrm{GW}}}{f^{4} \tau_{0}^{3}} \frac{M p T_{\mathrm{obs}}}{\delta t_{\mathrm{rms}}^{2}} d_{\text {near }} .
\end{aligned}
$$

We would now like to determine how large a $\mathrm{SNR}_{\text {near }}$ we could expect for a given $\Omega_{\mathrm{GW}}$, and for given observational parameters $M, p, T_{\mathrm{obs}}$, and $\delta t_{\mathrm{rms}}^{2}$. This amounts to maximizing $\mathrm{SNR}_{\text {near }}$ with respect to the GW-source parameters $V_{R}$, $T_{R}$, and $T_{\text {sig }}$; since these appear together in $d_{\text {near }}$, we obtain the largest possible $\mathrm{SNR}_{\text {near }}$ by setting $d_{\text {near }}=\tau_{0}$, the Hubble distance. We dare not place the GW source farther, since we are considering the "local" Universe and neglecting redshift effects.

Note that the scaling $\mathrm{SNR}_{\text {near }}^{2} \propto d_{\text {near }}$ of Eq. (5.7) seems counterintuitive, since we would naively think of the strongest sources as the closest. However, while the squared GW strain $h^{2}$ at the Earth scales as $1 / d^{2}$, it also scales with the total energy $\Delta E$ that is emitted by each source, and that is "available" to each source given a fixed $\Omega_{\mathrm{GW}}$; this $\Delta E$ increases with decreasing source density, and is proportional to $d_{\text {near }}^{3}$. This surprising intermediate result was already shown in ZT82 [22].
We can now plug in fiducial values for the observational parameters (as well as $\tau_{0}=3 \times 10^{17} \mathrm{~s}$ ), arriving at

$$
\begin{aligned}
\max \{\mathrm{SNR}\} & \lesssim 10\left(\frac{f}{10^{-7} \mathrm{~Hz}}\right)^{-2}\left[\frac{\Omega_{\mathrm{GW}}}{10^{-5}}\right]^{1 / 2} \times \text { obs. } \\
& \lesssim 0.03\left(\frac{f}{10^{-5} \mathrm{~Hz}}\right)^{-2}\left[\frac{\Omega_{\mathrm{GW}}}{10^{-2}}\right]^{1 / 2} \times \text { obs. }
\end{aligned}
$$

where

$$
\text { obs. }=\left[\frac{\delta t_{\mathrm{rms}}}{10^{-7} \mathrm{~s}}\right]^{-1}\left[\frac{M p T_{\mathrm{obs}}}{10^{4}}\right]^{1 / 2} \text {. }
$$

While we derived these constraints for the case of small $z$, we shall see below that they become even stronger for high- $z$ sources.

The fiducial values for $f$ and $\Omega_{\mathrm{GW}}$ in the second row of Eq. (5.8) are motivated by our original question, whether PTA searches should be extended to frequencies as high as $\sim 10^{-5} \mathrm{~Hz}$, and by the cosmological constraint (mentioned above) that $\Omega_{\mathrm{GW}} \lesssim 6 \times 10^{-3}$ [44]. Our conclusion is that a PTA detection of GWs at frequencies above $\sim 10^{-5} \mathrm{~Hz}$ should be considered very unlikely on fundamental grounds.

\section{B. Discovery space for unmodeled GW bursts in the low-redshift Universe}

Quite simply, a burst is a signal with $T_{\text {sig }} \sim 1 / f$. Since it contains only $\sim 1$ cycles, its instantaneous SNR (i.e., GW amplitude over rms noise) is the same as its matchedfiltering SNR, up to a factor of order 1 (after the data has been filtered to remove the noise that is outside the band of interest). Now, whatever the $T_{\text {sig }}$, we can still adjust $R_{4}$ so that $d_{\text {near }}$, as defined in Eq. (5.6), is equal to $\tau_{0}$.

For instance, if $T_{\text {sig }} \lesssim T_{\text {obs }}$ and $T_{\text {obs }}=10^{8} \mathrm{~s}$, this requires one burst every $10^{8} \mathrm{~s}$ within a Hubble volume. So for this rate, the instantaneous SNR is the same as given in Eq. (5.8) for modeled signals. This seems promising, because since bursts require no model for their detection, they could potentially reveal phenomena that nobody ever thought of. At the same time, their detection would require the utmost care in excluding instrumental and astrophysical artifacts.

\section{Discovery space for GW memory in the low-redshift Universe}

GWs with memory (for a recent review see [48]) cause a permanent deformation-a "memory" of the passage of the waves - in the configuration of an idealized GW detector. They are emitted by systems with unbound components (linear memory), and by generic GW sources because of the contribution of the energy momentum of their "standard" GWs to the changing radiative moments of the source (nonlinear memory). Several authors have 
discussed the detectability of the GW memory effect by PTAs for known source types, especially merging massiveblack-hole binaries $[17,18,49,50]$. Here we consider the effect from the point of view of the PTA discovery space, and again we ask in which region of parameter space PTAs could discover previously unimagined sources by way of their GW memory.

For a source at distance $d$ from Earth, which emits a total energy of $\Delta E$ in GWs, the amplitude of the memory effect is [17]

$$
h_{\mathrm{mem}} \sim \frac{\alpha}{\sqrt{6}} \frac{\Delta E}{d},
$$

where $\alpha$ is a factor determined by the asphericity of the energy outflow (more precisely, from its quadrupolar part). For coalescing binaries (a case of moderate beaming), $\alpha \approx 1$ [17], and $\alpha$ remains of order 1 even for extreme beaming.

In addition to the general assumptions we made in Sec. V, we will postulate that most of the GW energy from any one source is emitted on a timescale $T_{\text {sig }} \ll T_{\text {obs }}$. Then we can approximate the "turn on" of the memory effect as a step function, and the effect on any pulsar is to create a timing residual that grows linearly in time:

$$
\delta t_{\mathrm{GW}} \sim h_{\mathrm{mem}} \theta\left(t-t_{0}\right)\left(t-t_{0}\right),
$$

where the memory passes over the Earth at time $t_{0}$.

In any single pulsar, a linear-in-time residual can be interpreted simply as a glitch causing an instantaneous change in the pulsar frequency. However, all pulsars in the PTA would show such apparent glitches at the same time, with relative amplitudes following a simple pattern on the sky [17] determined by four parameters (the sky-location angles and two amplitudes that specify the transverse-trace-free part of the metric), so in principle the detection problem is well posed. The corresponding PTA SNR is [17]

$$
\mathrm{SNR}_{\mathrm{mem}} \sim \frac{1}{20} \frac{h_{\mathrm{mem}} T_{\mathrm{obs}}}{\delta t_{\mathrm{rms}}}\left(M p T_{\mathrm{obs}}\right)^{1 / 2},
$$

where the factor $1 / 20$ accounts for the facts that $\delta t_{\mathrm{GW}}$ will typically be zero for a significant fraction of $T_{\mathrm{obs}}$, and that a large part of the effect will be absorbed in the pulsars' timing models (and especially by the fitting of their periods and period derivatives) [17]. Note that GW memory effect is essentially a low-frequency effect: SNR can build up precisely because memory remains constant, but nonzero, for a sizable fraction of $T_{\text {obs }}$. Thus there is no particular advantage to high-cadence timing measurements.

We can now derive how large a SNR we may expect for detecting GW memory for a given $\Omega_{\mathrm{GW}}$ and for given observational parameters. As above, we relate the energy density in GWs to the energy emitted in GW bursts,

$$
\Omega_{\mathrm{GW}} \sim 10 \Delta E R_{4} \tau_{0}^{3}
$$

we then combine Eqs. (5.10), (5.12), and set $d=d_{\text {near }}=$ $\left(4 \pi R_{4} T_{\mathrm{obs}} / 3\right)^{-1 / 3}$, to obtain

$$
\mathrm{SNR}_{\text {mem,near }} \sim \frac{\alpha}{300} \frac{\Omega_{\mathrm{GW}}}{\tau_{0}^{3}} R_{4}^{-2 / 3} T_{\mathrm{obs}}^{4 / 3} \frac{\left(M p T_{\mathrm{obs}}\right)^{1 / 2}}{\delta t_{\mathrm{rms}}} .
$$

Again, for fixed $\Omega_{\mathrm{GW}}$ we maximize $\mathrm{SNR}_{\text {mem,near }}$ by taking $R_{4}$ to be as small as possible, subject to the constraint that $d_{\text {near }}<\tau_{0}$, leading to

$$
\begin{aligned}
\max \left\{\mathrm{SNR}_{\mathrm{mem}}\right\} & \simeq \frac{\alpha}{500} \frac{\Omega_{\mathrm{GW}}}{\tau_{0}} T_{\mathrm{obs}}^{2} \frac{\left(M p T_{\mathrm{obs}}\right)^{1 / 2}}{\delta t_{\mathrm{rms}}} \\
& \simeq 700 \alpha\left[\frac{\Omega_{\mathrm{GW}}}{10^{-2}}\right]\left[\frac{T_{\mathrm{obs}}}{10^{8} \mathrm{~s}}\right]^{2} \times \text { obs. }
\end{aligned}
$$

Comparing Eqs. (5.8) and (5.15), we see that—depending on the values of $\Omega_{\mathrm{GW}}$ and $f$ - the memory effect from a burst could be much more detectable than its direct waves. More generally, comparing $\mathrm{SNR}_{\text {mem }}$ with the direct $\mathrm{SNR}$ for the same source, as given by Eqs. (5.1) and (5.2), we find

$$
\begin{aligned}
\frac{\mathrm{SNR}_{\mathrm{mem}}}{\mathrm{SNR}_{\mathrm{dir}}} & =\frac{1 / 20}{1 / 20} \frac{h_{\mathrm{mem}} T_{\mathrm{obs}}}{h / f}\left(\frac{M p T_{\mathrm{obs}}}{M p T_{\mathrm{sig}}}\right)^{1 / 2} \\
& =\frac{1 / 20}{1 / 20} \frac{\pi^{2} \alpha}{2 \sqrt{6}} h f^{3} d T_{\mathrm{sig}} T_{\mathrm{obs}}\left(\frac{M p T_{\mathrm{obs}}}{M p T_{\mathrm{sig}}}\right)^{1 / 2} \\
& =\frac{1}{1 / 20} \frac{\pi^{2} \alpha}{2 \sqrt{6}} \mathrm{SNR}_{\mathrm{dir}} \frac{\delta t_{\mathrm{rms}} T_{\mathrm{sig}}^{-4} T_{\mathrm{obs}}^{2}}{\left(M p T_{\mathrm{obs}}\right)^{1 / 2}} \\
& \simeq 10^{2} \alpha \mathrm{SNR}_{\mathrm{dir}}\left[\frac{T_{\mathrm{sig}}}{10^{6} \mathrm{~s}}\right]^{-4}\left[\frac{T_{\mathrm{obs}}}{10^{8} \mathrm{~s}}\right]^{2}\left[\frac{d}{\tau_{0}}\right][\mathrm{obs} .]^{-1},
\end{aligned}
$$

where in the second row we have used the fact that $h_{\mathrm{mem}} \simeq(\alpha / \sqrt{6})(\Delta E / d)$ and $\Delta E=\left(\pi^{2} / 2\right) h^{2} f^{2} d^{2} \times T_{\text {sig }}$; in the third row we have substituted $\mathrm{SNR}_{\text {dir }}=$ $(1 / 20)(h / f) \delta t_{\text {rms }}^{-1}\left(M p T_{\text {sig }}\right)^{1 / 2}$ and replaced $f$ with $1 / T_{\text {sig }}$, as appropriate for a burst signal. Since $\mathrm{SNR}_{\mathrm{dir}}$ scales as $h_{\text {dir }}$ while $\mathrm{SNR}_{\text {mem }}$ scales as $h_{\text {dir }}^{2}$, the memory effect dominates for a sufficiently strong signal.

\section{DISCOVERY SPACE AT HIGH REDSHIFT}

In the previous section we have considered sources at small $z$, neglecting cosmological effects. We now turn to sources in the early Universe, at $z \gg 1$. Again, we will assume that the sources are isotropically distributed and that the Earth does not have a preferred location in spacetime with respect to them. The especially interesting cases are GW memory, which we discuss first, and unmodeled bursts.

We begin by collecting a few useful formulas. Let $t \equiv \int a^{-1}(\tau) \mathrm{d} \tau$ be the conformal time coordinate, in terms of which the (spatially flat) Robertson-Walker metric becomes 


$$
d s^{2}=a^{2}(t)\left[-d t^{2}+d x^{2}+d y^{2}+d z^{2}\right] .
$$

We find it useful to divide the high- $z$ epoch into the radiation-dominated era for $z \ll z_{\mathrm{eq}}$ and the matter-dominated era for $z \gg z_{\text {eq }}$, where $z_{\text {eq }} \approx 3,200$ (the redshift at which the energies of matter and radiation were equal). Then we can approximate $a(\tau) \propto \tau^{1 / 2}$ for $\tau<\tau_{\text {eq }}$ and $a(\tau) \propto \tau^{2 / 3}$ for $\tau>\tau_{\text {eq }}$ (of course, we now know that the Universe is dark energy, rather than matter dominated for $z \lesssim 1.7$, but we neglect this correction in keeping with the backof-the-envelope spirit of this paper).

We use the subscript " 0 " to refer to present Universe (e.g., $\tau_{0} \sim 10^{10}$ years is the present age of the Universe), and we choose our spatial coordinates so that $a_{0} \equiv a\left(\tau_{0}\right)=1$. Then

$$
t(z)= \begin{cases}\left(1+z_{\mathrm{eq}}\right)\left(3 \tau_{\mathrm{eq}}^{2 / 3} \tau^{1 / 3}(z)-\tau_{\mathrm{eq}}\right) & z<z_{\mathrm{eq}}, \\ \left(1+z_{\mathrm{eq}}\right)\left(2 \tau_{\mathrm{eq}}^{1 / 2} \tau^{1 / 2}(z)\right) & z>z_{\mathrm{eq}},\end{cases}
$$

and in particular,

$$
t_{0} \simeq\left(1+z_{\mathrm{eq}}\right)\left(3 \tau_{\mathrm{eq}}^{2 / 3} \tau_{0}^{1 / 3}\right),
$$

and therefore

$$
\frac{t_{0}}{t(z)} \simeq \begin{cases}(1+z)^{1 / 2} & z<z_{\mathrm{eq}}, \\ \frac{3}{2}(1+z)\left(1+z_{\mathrm{eq}}\right)^{-1 / 2} & z>z_{\mathrm{eq}} .\end{cases}
$$

Now consider GW bursts produced at $z \gg 1$. The size of the particle horizon at redshift $z$ is $\sim t(z)$ in comoving coordinates, and so the number of such particle-horizon volumes within our horizon volume today is $\sim\left[t_{0} / t(z)\right]^{3}$. Let $B$ be the average number of GW bursts coming from each horizon volume $[t(z)]^{3}$. Let the energy (as measured at $z$ ) of a typical burst be $\Delta E(z)$; by today that energy has been redshifted to $\Delta E_{0}=\Delta E_{z} /(1+z)$. The total energy today, within a Hubble volume, from all such bursts at redshift $z$ is $\Delta E_{0} B\left[t_{0} / t(z)\right]^{3}$, and it satisfies

$$
\Delta E_{0} B\left[t_{0} / t(z)\right]^{3} \lesssim \frac{1}{10} \Omega_{\mathrm{GW}} \tau_{0}
$$

We write "§" instead of " $\simeq$ " because there could be other significant sources for $\Omega_{\mathrm{GW}}$, besides this early-Universe contribution.

\section{A. Discovery space for GW memory from sources at high $z$}

The generalization of Eq. (5.10) to sources at arbitrary $z$ is

$$
h_{\mathrm{mem}} \sim \frac{\alpha}{\sqrt{6}} \frac{\Delta E(z)(1+z)}{D_{L}},
$$

where $\Delta E(z)$ is the locally measured energy loss and $D_{L}$ is the luminosity distance to the source (this follows from the propagation of GW-like perturbations in the Robertson-Walker spacetime [25] and from the definition of $D_{L}$ ). The energy carried by those emitted waves today is $\Delta E_{0}=\Delta E(z) /(1+z)$, while for high $z$ we have $D_{L} \approx 3 \tau_{0}(1+z)$. Thus we have

$$
h_{\mathrm{mem}} \simeq \frac{\alpha}{8} \frac{\Delta E_{0}(1+z)}{\tau_{0}} .
$$

It is instructive to determine the high- $z$ version of Eq. (5.16) for the ratio $\mathrm{SNR}_{\mathrm{mem}} / \mathrm{SNR}_{\mathrm{dir}}$. The only change in the derivation is the replacement $d \rightarrow 3 \tau_{0}(1+z)$, leading to

$$
\begin{aligned}
\frac{\mathrm{SNR}_{\text {mem }}}{\mathrm{SNR}_{\text {dir }}} \simeq & 3 \times 10^{13} \alpha \mathrm{SNR}_{\mathrm{dir}} \\
& \times\left[\frac{1+z}{10^{7}}\right]\left[\frac{T_{\text {sig }}}{10^{5} \mathrm{~s}}\right]^{-4}\left[\frac{T_{\text {obs }}}{10^{8} \mathrm{~s}}\right]^{2}\left[\frac{d}{\tau_{0}}\right][\text { obs. }]^{-1} .
\end{aligned}
$$

By combining Eqs. (6.4), (6.5), and (6.7), we can constrain $\mathrm{SNR}_{\mathrm{mem}}$ given $B$ and $\Omega_{\mathrm{GW}}$ :

$$
h_{\mathrm{mem}} \lesssim \frac{\alpha}{80} \frac{\Omega_{\mathrm{GW}}}{B} \times\left\{\begin{array}{cc}
\frac{1}{(1+z)^{1 / 2}} & 1 \ll z \ll z_{\mathrm{eq}}, \\
\frac{\left(1+z_{\mathrm{eq}}\right)^{3 / 2}}{3(1+z)^{2}} & z \gg z_{\mathrm{eq}}
\end{array}\right.
$$

the corresponding SNR follows from Eq. (5.12). We want to have a high probability of seeing one such signal within the observation time $T_{\text {obs }}$. Since the local rate can be shown ${ }^{2}$ to be $R \sim 4 \pi\left(B / \tau_{0}\right)\left[t_{0} / t(z)\right]^{3}$. Imposing $R T_{\text {obs }} \gtrsim$ 1 leads to

$$
\begin{aligned}
\max \left\{\mathrm{SNR}_{\mathrm{mem}}\right\} & \simeq \frac{\alpha}{125}(1+z) \frac{\Omega_{\mathrm{GW}}}{\tau_{0}} T_{\mathrm{obs}}^{2} \frac{\left(M p T_{\mathrm{obs}}\right)^{1 / 2}}{\delta t_{\mathrm{rms}}} \\
& \simeq 270 \alpha\left[\frac{1+z}{10^{7}}\right]\left[\frac{\Omega_{\mathrm{GW}}}{10^{-10}}\right]\left[\frac{T_{\mathrm{obs}}}{10^{8} \mathrm{~s}}\right]^{2} \times \text { obs. },
\end{aligned}
$$

a factor of order $(1+z)$ larger than the limit we derived in Eq. (5.15) for sources at $z \lesssim 1$. We regard this as a promising result, since current constraints on $\Omega_{\mathrm{GW}}$ still leave a great deal of room for possible discovery.

\section{B. Discovery space for unmodeled GW bursts at high $z$}

We now examine the prospects for detecting a GW burst from high $z$. The total energy emitted by such a source is

\footnotetext{
${ }^{2}$ Briefly, this can be shown by using Eq. (10) of [28], approximating the term $4 \pi\left(a_{0} r_{1}\right)^{2} \equiv 4 \pi\left(a_{0}\left(t_{0}-t(z)\right)\right)^{2}$ by $4 \pi\left(a_{0} t_{0}\right)^{2} \equiv 4 \pi\left(\tau_{0}\right)^{2}$ and using $\dot{n}(z)\left(d \tau_{1} / d z\right) \Delta z=\dot{n}(z) \Delta \tau_{1}=$ $\left(B / \tau_{0}^{3}\right)\left(t_{0} / t(z)\right)^{3}$.
} 


$$
\Delta E(z)=\Delta E_{0}(1+z) \simeq \frac{\pi^{2}}{2} h^{2} f^{2} T_{\operatorname{sig}} D_{L}^{2} ;
$$

using Eq. (6.5) and $D_{L} \sim 3 \tau_{0}(1+z)$, we then have

$$
\begin{aligned}
h^{2} \lesssim & 2 \times 10^{-3} \frac{\Omega_{\mathrm{GW}}}{B}\left(f \tau_{0}\right)^{-1}\left(f T_{\mathrm{sig}}\right)^{-1} \\
& \times\left\{\begin{array}{lc}
(1+z)^{-5 / 2} & 1 \ll z \ll z_{\mathrm{eq}}, \\
(1 / 3)\left(1+z_{\mathrm{eq}}\right)^{3 / 2}(1+z)^{-4} & z \gg z_{\mathrm{eq}} .
\end{array}\right.
\end{aligned}
$$

Again, a high probability of observing a signal constrains the rate $R$ according to $R \max \left\{T_{\text {sig }}, T_{\text {obs }}\right\} \gtrsim 1$, leading to

$$
\begin{aligned}
\max \left\{\mathrm{SNR}_{\mathrm{dir}}\right\} & \lesssim \frac{1}{120}\left[\frac{\Omega_{\mathrm{GW}}}{1+z}\right]^{1 / 2} \frac{\left(M p T_{\mathrm{obs}}\right)^{1 / 2}}{\left(f \delta t_{\mathrm{rms}}\right)\left(f \tau_{0}\right)} \\
& \approx 10\left[\frac{f}{10^{-7} \mathrm{~Hz}}\right]^{-2}\left[\frac{\Omega_{\mathrm{GW}}}{10^{-5}(1+z)}\right]^{1 / 2} \times \text { obs. }
\end{aligned}
$$

This is basically the same limit we found for the largest-SNR burst at $z<1$, but multiplied by the factor $(1+z)^{-1 / 2}$.

\section{CORRECTIONS FOR BEAMING AND FOR GALACTIC SOURCES}

So far our estimates of signal strengths have implicitly assumed that the radiation is not strongly beamed. We have also implicitly assumed that detectable PTA sources will be extra-Galactic. In this section we briefly show how our estimates get modified if one drops these assumptions. Both these issues were addressed by ZT82 [22], but here we extend their considerations to large $z$.

\section{A. Modifications for highly beamed radiation}

Assume that the GW energy is beamed into solid angle $4 \pi F$. To see how $\max \{\mathrm{SNR}\}$ for "direct" radiation scales with $F$, we will take $\Omega_{\mathrm{GW}}$ and the total radiated energy to be fixed, which together imply a fixed rate density. For the case $z \lesssim 1$, we can approximate space as Euclidean, so the distance $d$ to the closest source beaming in our direction scales as $d \propto F^{-1 / 3}$; the observed $h$ scales as $h \propto F^{-1 / 2} / d$; and altogether $h \propto F^{-1 / 6}$. We see that the effect of beaming on $\max \{\mathrm{SNR}\}$ is extremely weak; for instance, a beaming factor $F=10^{-3}$ yields only a factor $\sim 3$ increase in the potential SNR. This very weak dependence was already noted by ZT82 in the $z \lesssim 3$ case.

For $z \gg 1$, to account for beaming, on the right-hand side of Eq. (6.11) we would replace $\Delta E_{0}$ with $\Delta E_{0} / F$. However the condition $R T_{\text {obs }} \gtrsim 1$ gets replaced by $R F T_{\mathrm{obs}} \gtrsim 1$, which leads to $\Delta E_{0} \propto \Omega_{\mathrm{GW}} B^{-1} F$. Thus the $F$ factors cancel, and beaming has basically no effect on $\max \{\mathrm{SNR}\}$ for high $-z$ sources. Note that our low $-z$ and high- $z$ upper limits, Eqs. (5.8) and (6.13) respectively, have slightly different character: for the former we maximize the SNR from the nearest detected source, for the latter we fix $z$ and therefore luminosity distance under the constraint of detecting at least one source during the experiment.

What about memory? The effect of beaming is negligible, since the memory component of GW strain is not beamed, even when direct waves are. The dominant effect is that the parameter $\alpha$ changes by a factor of order $1 \mathrm{com}-$ pared to the case of quadrupole emission.

\section{B. Modifications for Galactic sources}

Throughout Secs. V and VI we have assumed that the Earth does not occupy a preferred location in the Universe. However the Earth lies in the Galaxy; how might that modify our results? For sources in the low- $z$ Universe, we showed in Sec. V A that, for fixed $\Omega_{\mathrm{GW}}$, detection SNR is maximized for sources whose event rate is once per $T_{\text {obs }}$ in a Hubble volume. For a Galactic source to be observable, this rate must increase to once per $T_{\text {obs }}$ per Milky-Way-like galaxy, or $\sim 10^{9}$ times greater. To maintain the same $\Omega_{\mathrm{GW}}$, the energy $\Delta E$ radiated per event must decrease by a factor $10^{9}$. (We must also assume that the Galaxy can sustain such a rate of events.) On the other hand, the distance to the extragalactic source is $\sim 3 \mathrm{Gpc}$, compared to $\sim 10 \mathrm{kpc}$ for a randomly located Galactic source. For the direct radiation, $h \propto \Delta E^{1 / 2} / d$, so the ratio

$$
\frac{\max \left\{\mathrm{SNR}_{\mathrm{dir}}^{\mathrm{Gal}}\right\}}{\max \left\{\mathrm{SNR}_{\mathrm{dir}}^{z \sim 1}\right\}} \sim 10^{-9 / 2} \frac{3 \mathrm{Gpc}}{10 \mathrm{kpc}} \sim 10,
$$

as was first shown by ZT82 [22]. Thus, besides being intrinsically less plausible, putative Galactic sources increase $\max \left\{\mathrm{SNR}_{\text {dir }}\right\}$ by only an order of magnitude, compared to the $z \sim 1$ case.

While we have undertaken the above calculation in the spirit of completeness, we point out that to account for an overall $\Omega_{\mathrm{GW}} \sim 10^{-2}$ (say), these putative Galactic explosions would have to release $\sim 50 M_{\odot}$ in GW energy roughly every $\sim 3 \mathrm{yr}$, and it would appear difficult to construct a plausible physical mechanism for such explosions that would not already have been detected by other means.

For the memory effect, $h \propto \Delta E / d$, so we may estimate a ratio

$$
\frac{\max \left\{\mathrm{SNR}_{\mathrm{mem}}^{\mathrm{Gal}}\right\}}{\max \left\{\mathrm{SNR}_{\mathrm{mem}}^{z \sim 1}\right\}} \sim 10^{-9 / 2} \frac{3 \mathrm{Gpc}}{10 \mathrm{kpc}} \sim 10^{-3.5} .
$$

Finally, we note that if we had focused on sources in the Local Group instead of just the Milky Way, the event rate for sources outside the Milky Way would be dominated by Andromeda. Since Andromeda has roughly the same mass as the Milky Way but is $\sim 100$ times further away than our Galactic center, the strongest such events would be $\sim 100$ times weaker than Galactic events. 


\section{Conclusions and Caveats}

In the paper we have constrained and characterized the GW discovery space of PTAs on the basis of energetic and statistical considerations alone. In Secs. V and VI we showed that a PTA detection of GWs at frequencies above $\sim 10^{-5} \mathrm{~Hz}$ would either be an extraordinary coincidence, or have extraordinary implications; this effect results from an analysis of fundamental constraints on possible sources across the PTA sensitivity range, rather than deficiencies in PTA detection itself. We showed also that GW memory can be more detectable than direct GWs, and that memory increasingly dominates the total SNR of an event for sources at higher and higher redshifts; indeed, GW memory from high $-z$ sources represents a large discovery space for PTAs.

Although we assumed modest beaming in our estimates, in Sec. VII we argued that even extreme beaming would have a minor impact on detection SNRs. Similarly, although we assumed that the strongest GW sources during PTA observation would be extragalactic, our constraint on $\max \{\mathrm{SNR}\}$ rises only by a factor $\sim 10$ for Galactic sources. Throughout the paper we adopted a SNR scaling law valid for white pulsar noise; in Sec. II we explained, on the basis of toy model and of the observational characterization of pulsar noise, why this was appropriate.

In Sec. IV we demonstrated how to properly incorporate the effects of red noise in PTA searches, and we demonstrated that the effects of periodic GWs between $\sim 10^{-7}$ and $10^{-4.5} \mathrm{~Hz}$ band would not be degenerate with small errors in the standard pulsar parameters, except in a few very narrow bands.

Theoretical upper limits are akin to no-go theorems, and the authors are well aware that the history of the latter in physics is replete with examples of results that, while strictly correct, turned out to be misleading because their assumptions were overly restrictive. For this reason, our chief motivation in doing this research was not to rule out possibilities, but to uncover promising but neglected areas of search space. With this in mind, we now recall some of the assumptions that we have made, and point out some of the ways that nature could be side stepping them.

(i) In this paper we assumed that the Earth is not in a preferred location in the Universe. In Sec. VII we considered the case in which relevant $\mathrm{GW}$ sources are clustered in galaxies, but still assumed that the Earth is not in some preferred location within the Milky Way.

(ii) Even if the Earth does not occupy a preferred location with respect to relevant GW sources, some millisecond pulsars might do so. For instance, if two or more pulsars are located in a globular cluster that also contains a BH binary with masses $\gtrsim 1000 M_{\odot}$ the correlated timing residuals due to the binary's GWs impinging on the pulsars could well be detectable (see, e.g., [51]).

(iii) In this paper we assumed that at any redshift $z$ there are no structures (such as phase-transition bubbles) that are significantly larger than the contemporaneous horizon size $t(z)$. This is a reasonable way to incorporate causality constraints for processes that are not correlated on superhorizon scales to begin with, but it certainly does not hold for all cases: for instance, inflation would imprint correlations on much larger scales. So a priori there could arise strong GW sources that violate this assumption.

It might be worthwhile to try to come up with reasonable physical scenarios that violate one or more of our assumptions.

\section{ACKNOWLEDGMENTS}

C. C. gratefully acknowledges support from NSF Grant No. PHY-1068881. M. V. is grateful for support from the JPL RTD program. This work was carried out at the Jet Propulsion Laboratory, California Institute of Technology, under contract to the National Aeronautics and Space Administration.
[1] M. V. Sazhin, Sov. Astron. 22, 36 (1978).

[2] S. Detweiler, Astrophys. J. 234, 1100 (1979).

[3] R. W. Hellings and G. S. Downs, Astrophys. J. Lett. 265, L39 (1983).

[4] R. van Haasteren, Y. Levin, G. H. Janssen, K. Lazaridis, M. Kramer, B. W. Stappers, G. Desvignes, M. B. Purver, A. G. Lyne, R. D. Ferdman et al., Mon. Not. R. Astron. Soc. 414, 3117 (2011).

[5] M. Kramer and D. J. Champion, Classical Quantum Gravity 30, 224009 (2013).

[6] P. B. Demorest, R. D. Ferdman, M. E. Gonzalez, D. Nice, S. Ransom, I. H. Stairs, Z. Arzoumanian, A. Brazier, S. BurkeSpolaor, S. J. Chamberlin et al., Astrophys. J. 762, 94 (2013).
[7] M. A. McLaughlin, Classical Quantum Gravity 30, 224008 (2013).

[8] R. N. Manchester, G. Hobbs, M. Bailes, W. A. Coles, W. van Straten, M. J. Keith, R. M. Shannon, N. D. R. Bhat, A. Brown, S. G. Burke-Spolaor et al., Pub. Astron. Soc. Aust. 30, e017 (2013).

[9] G. Hobbs, Classical Quantum Gravity 30, 224007 (2013).

[10] G. Hobbs, A. Archibald, Z. Arzoumanian, D. Backer, M. Bailes, N. D. R. Bhat, M. Burgay, S. Burke-Spolaor, D. Champion, I. Cognard et al., Classical Quantum Gravity 27, 084013 (2010).

[11] R. N. Manchester, Classical Quantum Gravity 30, 224010 (2013). 
[12] A. Sesana, Mon. Not. R. Astron. Soc. 433, 1 (2013).

[13] X. Siemens, J. Ellis, F. Jenet, and J. D. Romano, Classical Quantum Gravity 30, 224015 (2013).

[14] A. Sesana, A. Vecchio, and M. Volonteri, Mon. Not. R. Astron. Soc. 394, 2255 (2009).

[15] D. R. B. Yardley, G. B. Hobbs, F. A. Jenet, J. P. W. Verbiest, Z. L. Wen, R. N. Manchester, W. A. Coles, W. van Straten, M. Bailes, N. D. R. Bhat et al., Mon. Not. R. Astron. Soc. 407, 669 (2010).

[16] A. Sesana, arXiv:1307.4086.

[17] R. van Haasteren and Y. Levin, Mon. Not. R. Astron. Soc. 401, 2372 (2010).

[18] J. M. Cordes and F. A. Jenet, Astrophys. J. 752, 54 (2012).

[19] A. H. Jaffe and D. C. Backer, Astrophys. J.583, 616 (2003).

[20] R. M. Shannon, V. Ravi, W. A. Coles, G. Hobbs, M. J. Keith, R. N. Manchester, J. S. B. Wyithe, M. Bailes, N. D. R. Bhat, S. Burke-Spolaor et al., Science 342, 334 (2013).

[21] F. A. Jenet, G. B. Hobbs, K. J. Lee, and R. N. Manchester, Astrophys. J. Lett. 625, L123 (2005).

[22] M. Zimmermann and K. Thorne, in Essays in General Relativity: A Festschrift for Abraham Taub, edited by F. J. Tipler (Academic Press, New York, 1980), pp. 139.

[23] D. R. Lorimer, Living Rev. Relativity 11, 8 (2008), 0811.0762 .

[24] I. H. Stairs, Living Rev. Relativity 6, 5 (2003).

[25] M. Maggiore, Gravitational Waves: Volume 1: Theory and Experiments, Gravitational Waves (Oxford University Press, Oxford, UK, 2008).

[26] G. L. Bretthorst, AIP Conf. Proc. 567, 1 (2001).

[27] R. van Haasteren, Y. Levin, P. McDonald, and T. Lu, Mon. Not. R. Astron. Soc. 395, 1005 (2009).

[28] C. Cutler and J. Harms, Phys. Rev. D 73, 042001 (2006).

[29] W. W. Hager, SIAM Rev. 31, 221 (1989).

[30] J. M. Cordes and R. M. Shannon, arXiv:1010.3785.

[31] R. M. Shannon and J. M. Cordes, Astrophys. J.725, 1607 (2010).

[32] J. P. W. Verbiest, M. Bailes, W. A. Coles, G. B. Hobbs, W. van Straten, D. J. Champion, F. A. Jenet, R. N. Manchester,
N. D. R. Bhat, J. M. Sarkissian et al., Mon. Not. R. Astron. Soc. 400, 951 (2009).

[33] M. C. Begelman, R. D. Blandford, and M. J. Rees, Nature (London) 287, 307 (1980).

[34] J. S. B. Wyithe and A. Loeb, Astrophys. J.590, 691 (2003).

[35] S. T. McWilliams, J.P. Ostriker, and F. Pretorius, arXiv:1211.4590.

[36] J. Polchinski, arXiv:0707.0888.

[37] B. Allen, in Some Topics on General Relativity and Gravitational Radiation, edited by J. A. Miralles, J. A. Morales, and D. Saez (Edition Frontieres, Paris, 1997), p. 3.

[38] F. Dubath, J. Polchinski, and J. V. Rocha, Phys. Rev. D77, 123528 (2008).

[39] T. Damour and A. Vilenkin, Phys. Rev. D 64, 064008 (2001).

[40] T. Damour and A. Vilenkin, Phys. Rev. D71, 063510 (2005).

[41] B. P. Abbott, R. Abbott, F. Acernese, R. Adhikari, P. Ajith, B. Allen, G. Allen, M. Alshourbagy, R. S. Amin, S. B. Anderson et al., Nature (London) 460, 990 (2009).

[42] L. A. Boyle and A. Buonanno, Phys. Rev. D78, 043531 (2008).

[43] T. L. Smith, E. Pierpaoli, and M. Kamionkowski, Phys. Rev. Lett. 97, 021301 (2006).

[44] M. Archidiacono, S. Hannestad, A. Mirizzi, G. Raffelt, and Y. Y. Y. Wong, J. Cosmol. Astropart. Phys. 10 (2013) 020.

[45] J. A. Ellis, M. A. McLaughlin, and J. P. W. Verbiest, Mon. Not. R. Astron. Soc. 417, 2318 (2011).

[46] G. B. Hobbs, R. T. Edwards, and R. N. Manchester, Mon. Not. R. Astron. Soc. 369, 655 (2006).

[47] F. B. Estabrook and H. D. Wahlquist, Gen. Relativ. Gravit. 6, 439 (1975).

[48] M. Favata, Classical Quantum Gravity 27, 084036 (2010).

[49] N. Seto, Mon. Not. R. Astron. Soc. 400, L38 (2009).

[50] M. S. Pshirkov, D. Baskaran, and K. A. Postnov, Mon. Not. R. Astron. Soc. 402, 417 (2010).

[51] F. A. Jenet, T. Creighton, and A. Lommen, Astrophys. J. Lett. 627, L125 (2005). 